\title{
Detection of the protease codon 35 amino acid insertion in sequences from treatment-naïve HIV-1 subtype C infected individuals in the Central Region of Portugal
}

\author{
João Pereira-Vaz ${ }^{\mathrm{a}, *}$, Vitor Duque ${ }^{\mathrm{a}}$, Luís Trindade ${ }^{\mathrm{b}}$, \\ José Saraiva-da-Cunha ${ }^{c}$, António Meliço-Silvestre ${ }^{c}$ \\ a Laboratório de Virologia do Departamento de Doenças Infecciosas, Hospitais da Universidade de Coimbra, \\ Av Bissaya Barreto, 3000-075 Coimbra, Portugal \\ b Serviço de Doenças Infecciosas, Centro Hospitalar de Coimbra, Coimbra, Portugal \\ ${ }^{\mathrm{c}}$ Departamento de Doenças Infecciosas, Hospitais da Universidade de Coimbra, Coimbra, Portugal
}

\section{A R T I C L E I N F O}

\section{Article history:}

Received 30 March 2009

Received in revised form 23 June 2009

Accepted 23 June 2009

\section{Keywords:}

HIV-1

Subtype C

Protease

Insertion

\begin{abstract}
A B S T R A T
Background: Amino acids insertions in the protease (PR) coding region have been reported in protease inhibitors (PIs) treatment-naïve and experienced HIV-1 infected individuals ranging from $0.1 \%$ to $4.55 \%$ and have been rarely found in non-B HIV-1 subtype strains.

Objectives: To investigate the presence of amino acid insertions in the PR coding region in sequences from treatment-naïve HIV-1 infected individuals in the Central Region of Portugal.

Study design: Sequences of the pol gene from 260 treatment-naïve HIV-1 infected individuals between 2000 and 2008 were analyzed and phylogenetic analysis was performed.

Results: A threonine insertion (E35E_T) was detected in $2.69 \%(n=7)$ of the sequences analyzed and all the sequences that possessed this insertion were identified as subtype $C$. All the seven inserted sequences clustered in the same lineage of the phylogenetic tree. Heterosexual and intravenous drug use were found to be the routes of infection. No major mutations in the PR coding region associated with resistance to PIs were detected.

Conclusions: It was found the highest prevalence of PR codon 35 insertion among treatment-naïve HIV-1 infected individuals ever reported in the western countries. Epidemiological data and Phylogenetic analysis indicated the possibility of transmission of this insertion. The results suggested that these inserted strains have normal susceptibility to PIs containing regimens. This study demonstrated the spreading epidemic of PR codon 35 inserted strains from subtype $C$ in the Central Region of Portugal, during the past eight years.
\end{abstract}

(c) 2009 Elsevier B.V. All rights reserved.

\section{Background}

Human immunodeficiency virus type 1 (HIV-1) is characterized by extensive genetic variability caused by the high mutation rate of HIV-1 genome associated with the low fidelity of the reverse transcriptase (RT), the lack of proofreading function and the rapid turnover of viral population. ${ }^{1}$ A heterogeneous group of insertion mutations ranging from 3 to 18 nucleotides has been identified in HIV-1 strains at positions $17,19,22,25,31,33,35,36,37,70$, and 95 of the protease (PR) coding region. ${ }^{2-8}$ These insertion mutations are likely generated by the RT stalling and primer/template slippage that is also believed to generate RT insertion mutations. ${ }^{9,10}$ Amino acid insertions in the PR coding region have been reported

\footnotetext{
* Corresponding author. Tel.: +351 239400 595; fax: +351 239400595 .

E-mail address: joaovaz@huc.min-saude.pt (J. Pereira-Vaz).
}

in protease inhibitors (PIs) experienced and treatment-naïve HIV-1 infected individuals with prevalence ranging from $0.1 \%^{3}$ to $4.55 \%,{ }^{11}$ and have been rarely found in non-B HIV-1 subtype strains. ${ }^{6}$

It is still unclear the relative contributions of amino acid insertions in the PR coding region to the overall resistance to PIs and/or viral replication capacity. Amino acid insertions in the PR coding region have been reported not to modify the susceptibility to PIs, 3,12,13 However, a recent study reported that the amino acid insertions L33L_L and E35E_E contribute to the viral resistance to most of the tested PIs. ${ }^{8}$

\section{Objectives}

The objective of this study was to investigate the presence of amino acid insertions in the PR coding region in treatment-naïve HIV-1 infected individuals in the Central Region of Portugal. 


\section{Study design}

\subsection{Study population}

Available plasma samples from 260 treatment-naïve HIV-1 infected individuals between 2000 and 2008 were included in this study. Demographic, epidemiologic and clinical data was collected through a standardized protocol. Ethical approval was obtained from the two collecting centres as well as informed consent from the individuals.

\subsection{RNA extraction, reverse transcription, amplification and sequencing}

Viral RNA was extracted from plasma samples using the QIAamp Viral RNA Mini Kit (Qiagen). The PR and RT coding regions of the pol gene (1302 base pairs) were reverse transcribed, amplified and sequenced using the ViroSeq HIV-1 Genotyping System v2.0 (Celera Diagnostics) and an automated DNA sequencer, ABI Prism 3100 Genetic Analyzer (Applied Biosystems).

\subsection{Phylogenetic analysis and resistance mutations}

Phylogenetic analysis was carried out for HIV-1 genetic subtype identification using the neighbor-joining method. ${ }^{14}$ The pol nucleotide sequences and HIV-1 reference sequences obtained from the Los Alamos HIV database (http://hiv-web.lanl.gov) were aligned using ClustalX. ${ }^{15}$ PR codon 35 insertions were removed from nucleotide sequences prior to alignment. The pairwise distance matrix was estimated using the Kimura two-parameter model $^{16}$ and a transition/transversion ratio of 2.0 with the DNAdist program, as implemented in the PHYLIP software package (available at http://evolution.gs.washington.edu/phylip.html). Bootstrap re-sampling (1000 replicates) of the multiple alignment was performed to evaluate the reliability of the inferred tree. ${ }^{17}$

The prevalence of HIV-1 drug resistance associated mutations in the PR coding region was estimated according to the International AIDS Society mutation Table ${ }^{18}$ and by taking into account only major mutations in the PR coding region. Minor mutations in the PR coding region were not counted as molecular indicators associated with resistance given their high prevalence particularly for some of the HIV-1 non-B subtypes. The assessment of the impact on first line therapy response was done using the Stanford University HIV-1 genotypic resistance interpretation algorithm (http://hivdb.stanford.edu).

\subsection{Nucleotide sequence accession numbers}

The inserted nucleotide sequences of HIV-1 pol gene obtained in this study were submitted to GenBank and are available under the following accession numbers: FJ360877 (04PTHC_34), FJ360878 (04PTHC_38), FJ360879 (04PTHC_54), FJ360880 (04PTHC_60), FJ360881 (00PTHC_56), FJ360882 (08PTHC_17), and FJ360883 (08PTHC_84).

\section{Results}

A threonine (ACA) insertion (E35E_T) between codon 35 and 36 was detected in $2.69 \%(n=7)$ of the sequences analyzed $(n=260)$. All sequences that possessed the PR codon 35 insertion were identified as HIV- 1 subtype $C$, and represented $43.75 \%$ of the sequences identified as subtype $C(n=16)$ in this study. All 7 inserted isolates formed a cluster in the phylogenetic tree clearly bootstrap supported (bootstrap value of 1000), indicating their monophyletic origin (Fig. 1).

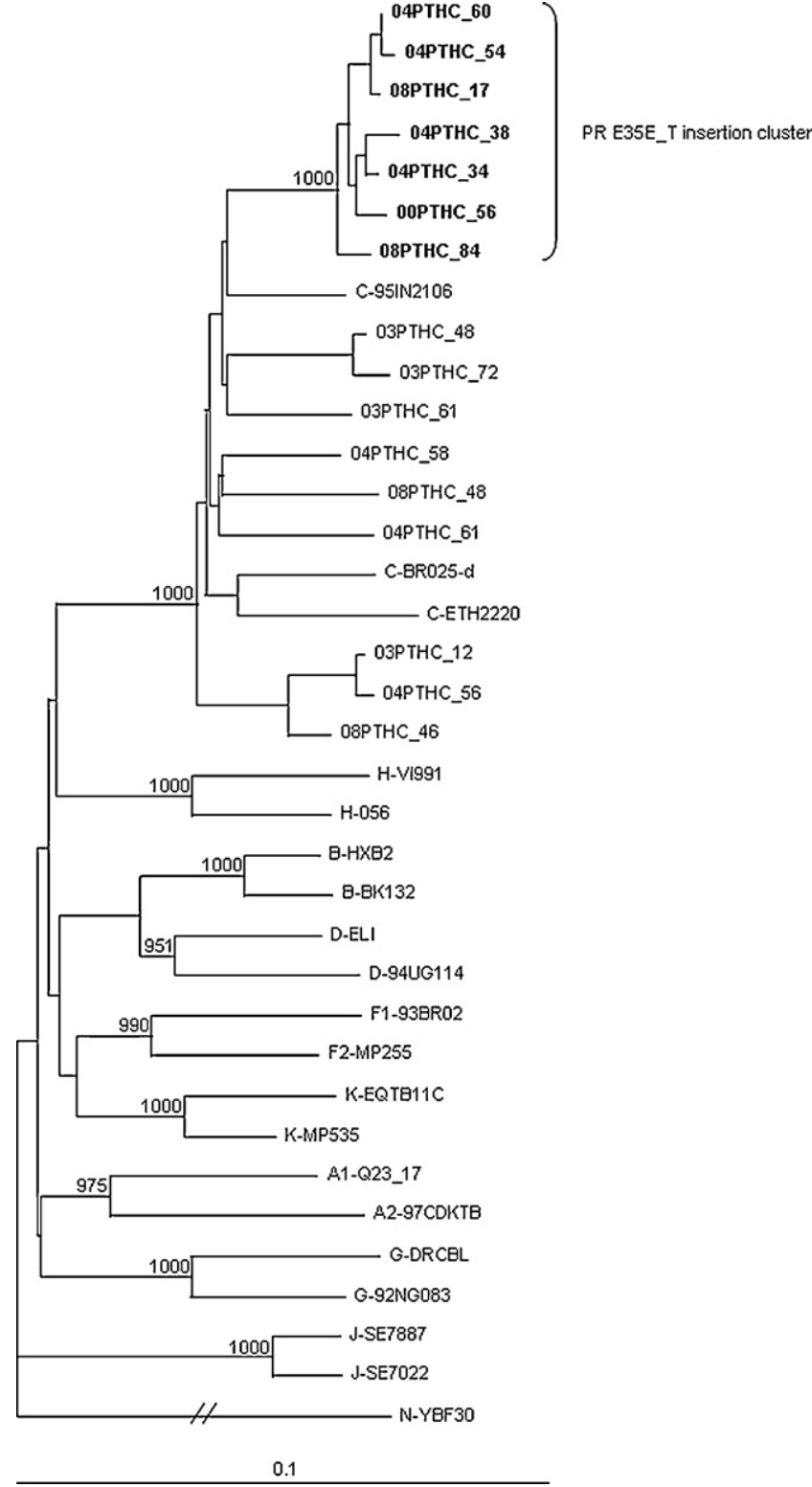

Fig. 1. Phylogenetic rooted Neighbor-joining tree with 1000 bootstrap replicates of the pol gene nucleotide sequences ( $1302 \mathrm{bp}$ ) of the isolates identified as subtype $\mathrm{C}$ in the Central Region of Portugal and HIV-1 reference sequences. Strain YBF30 (HIV-1 group N) was used as outgroup. The PR E35E_T insertion cluster is indicated and inserted isolates are shown in bold. Scale, genetic distances in 0.1 substitutions per nucleotide.

The epidemiological background revealed that these seven individuals carrying the inserted strains five were white males and two were white females. All individuals were resident in the city of Coimbra (Central Region of Portugal) and all reported that HIV-1 infection was probably acquired in this region indicating a possible epidemiological linkage between them. The female carrying the 08PTHC_84 strain admitted being a sexual worker. Three male individuals (00PTHC_56, 04PTHC_34 and 04PTHC_54) reported heterosexual risk behaviour with sexual workers from this region. However, only in one case was possible to clearly establish an epidemiological linkage where the female carrying the 04PTHC_38 strain reported unprotected sexual intercourse with the male carrying the 04PTHC_34 strain. Heterosexual (71.4\%; $n=5)$ and intravenous drug use $(28.6 \% ; n=2)$ transmission were found to be the routes of infection. These individuals had median HIV-1 RNA 
Table 1

Epidemiological characteristics of the HIV-1 infected individuals carrying the PR E35E_T inserted isolates.

\begin{tabular}{|c|c|c|c|c|c|c|c|}
\hline Individual & HIV-1 subtype & Gender & Country of infection & Transmission route & Sample collection date & $\begin{array}{l}\text { HIV-1 RNA viral load } \\
\text { (copies/ml) }\end{array}$ & $\mathrm{CD}^{+}\left(\right.$cells $\left./ \mathrm{mm}^{3}\right)$ \\
\hline 00РTHC_56 & $\mathrm{C}$ & Male & Portugal & Heterosexual & November 2000 & 5,110 & 418 \\
\hline 04PTHC_34 & $\mathrm{C}$ & Male & Portugal & Heterosexual & June 2004 & 2,980 & $--^{a}$ \\
\hline 04PTHC_38 & $\mathrm{C}$ & Female & Portugal & Heterosexual & May 2004 & $1,280,000$ & 68 \\
\hline 04PTHC_54 & $\mathrm{C}$ & Male & Portugal & Heterosexual & December 2004 & 19,000 & $--^{a}$ \\
\hline 04PTHC_60 & $\mathrm{C}$ & Male & Portugal & IVDU $^{\mathrm{b}}$ & November 2004 & 779,000 & 48 \\
\hline 08PTHC_17 & $\mathrm{C}$ & Male & Portugal & IVDU $^{\mathrm{b}}$ & January 2008 & 6,232 & 180 \\
\hline 08PTHC_84 & $\mathrm{C}$ & Female & Portugal & Heterosexual & May 2008 & $-{ }^{a}$ & $--^{a}$ \\
\hline
\end{tabular}

a Information not available.

b Intravenous drug use.

viral load and CD4 ${ }^{+}$count of 348,720 copies $/ \mathrm{ml}$ and 179 cells $/ \mathrm{mm}^{3}$, respectively (Table 1 ).

No major mutations in the PR coding region associated with resistance to PIs were detected in the nucleotide sequences from the seven inserted isolates. Two individuals carrying the E35E_T inserted strains started antiretroviral treatment with PIs containing regimens and had a normal virological response: the individual carrying the 08PTHC_17 strain started with lamivudine, zidovudine, and lopinavir/ritonavir, achieving HIV-1 RNA viral load $<50$ copies/ml by week 5; the individual carrying the 04PTHC_54 strain started with lamivudine, zidovudine, and indinavir, achieving HIV-1 RNA viral load $<50$ copies/ml by week 28 .

\section{Discussion}

It was found the highest prevalence (2.69\%) of the PR codon 35 insertion (E35E_T) among HIV-1 infected individuals ever reported in Europe ${ }^{8}$ and the United States. ${ }^{3,13}$ All the PR codon 35 inserted strains in the Central Region of Portugal were isolated from treatment-naïve subtype $C$ infected individuals while previous reports were associated mainly with PIs experienced HIV-1 infected individuals. 3,8,13 Furthermore, to our knowledge, this is the highest prevalence of amino acid insertions in the PR coding region ever found in HIV-1 non-B subtype strains. Since HIV-1 subtype C accounts for more than $50 \%$ of all infections worldwide, concentrated in regions (Southern and East Africa, and India) ${ }^{19}$ where genotyping in the monitoring of HIV-1 infection is not or poorly available, with increasing use of genotyping it could be expected that more PR inserted strains will be detected.

By combining epidemiological data of the individuals (Table 1) and the topologie of the phylogenetic tree (Fig. 1), this study demonstrated the heterosexual and intravenous drug use transmission of these inserted strains among hosts which is consistent with previously reported evidence of transmission between sexual partners. $^{2,11}$

The HIV-1 RNA viral load and $\mathrm{CD}^{+}$count of the seven individuals harboring the inserted isolates varied to a large extent (Table 1), suggesting that E35E_T insertion is not associated with clinical disease progression. However, the origin of this insertion mutation was not elucidated.

Two individuals started antiretroviral treatment with PIs containing regimens and achieved undetectable viral load levels, suggesting that the E35E_T insertion in the PR coding region does not contribute directly to PIs resistance which is in agreement with all ${ }^{2,3,13}$ except one, ${ }^{8}$ of the previous studies concerning insertion mutations at position 35. However, several observations $s^{3,8,13}$ suggest that insertion mutations at position 35 might confer an advantage in the replication capacity and survival of HIV-1 variant under selective drug pressure. Therefore, further investigation to characterize their effects on the viruses by studying PR resistance and replicative capacity and on the enzyme directly by using enzymological and structural analysis are needed.
In conclusion, our findings demonstrate the spreading epidemic of PR codon 35 inserted strains from subtype $C$ in the Central Region of Portugal, during the past eight years. For this reason it is important to know that this insertion mutation could be present before PIs treatment and is unlikely to influence treatment with these drugs.

\section{Conflict of interest}

None.

\section{References}

1. Roberts JD, Bebenek K, Kunkel TA. The accuracy of reverse transcriptase from HIV-1. Science 1988;242:1171-3.

2. Grant RM, Kahn JO, Wrin T, Drews B, Javier J, Webb M, et al. HIV-1 with an insertion in protease is drug-susceptible, replication-competent and transmissible. Antivir Ther 2001;6(Suppl. 1):44.

3. Kim EY, Winters MA, Kagan RM, Merigan TC. Functional correlates of insertion mutations in the protease gene of human immunodeficiency virus type 1 isolates from patients. J Virol 2001;75:11227-33.

4. Winters MA, Kagan RM, Heseltine PNR, Merigan TC. New two-amino acid insertion near codon 70 of the HIV type 1 protease gene. AIDS Res Hum Retroviruses 2005;21:311-3

5. Tramuto F, Bonura F, Mancuso S, Romano N, Vitale F. Detection of a new 3base pair insertion mutation in the protease gene of human immunodeficiency virus type 1 during highly active antiretroviral therapy (HAART). AIDS Res Hum Retroviruses 2005;21:420-3.

6. Bessong PO, Mphahlele J, Choge IA, Obi LC, Morris L, Hammarskjold ML, et al. Resistance mutational analysis of HIV type 1 subtype $C$ among rural South African drug-naive patients prior to large-scale availability of antiretrovirals. AIDS Res Hum Retroviruses 2006;22:1306-12.

7. Brann TW, Dewar RL, Jiang MK, Shah A, Nagashima K, MetcalfJA, et al. Functional correlation between a novel amino acid insertion at codon 19 in the protease of human immunodeficiency virus type 1 and polymorphism in the $\mathrm{p} 1 / \mathrm{p} 6 \mathrm{Gag}$ cleavage site in drug resistance and replication fitness. J Virol 2006;80:613645.

8. Kozísek M, Sasková KG, Rezácová P, Brynda J, van Maarseveen NM, De Jong D, et al. Ninety-nine is not enough: molecular characterization of inhibitor-resistant human immunodeficiency virus type 1 protease mutants with insertions in the flap region. J Virol 2008;82:5869-78.

9. Bebenek K, Abbotts J, Wilson SH, Kunkel TA. Error-prone polymerization by HIV-1 reverse transcriptase. Contribution of template-primer misalignment, miscoding, and termination probability to mutational hot spots. J Biol Chem 1993;268: $10324-34$

10. Harrison GP, Mayo MS, Hunter E, Lever AM. Pausing of reverse transcriptase on retroviral RNA templates is influenced by secondary structures both $5^{\prime}$ and $3^{\prime}$ of the catalytic site. Nucleic Acids Res 1998;26:3433-42.

11. Chen JHK, Wong KH, Chan KC, Lam HY, Yuen KY, Cheng VCC, et al. Molecular epidemiology and divergence of HIV type 1 protease codon 35 inserted strains among treatment-naive patients in Hong Kong. AIDS Res Hum Retroviruses 2008;24:537-42.

12. Stürmer M, Staszewski S, Doerr HW, Hertogs K. A 6-base pair insertion in the protease gene of HIV type 1 detected in a protease inhibitor-naive patient is not associated with indinavir treatment failure. AIDS Res Hum Retroviruses 2003;19:967-8.

13. Paolucci S, Baldanti F, Dossena L, Gerna G. Amino acid insertions at position 35 of HIV-1 protease interfere with virus replication without modifying antiviral drug susceptibility. Antiviral Res 2006;69:181-5.

14. Saitou N, Nei M. The neighbor-joining method: a new method for reconstructing phylogenetic trees. Mol Biol Evol 1987;4:406-25.

15. Thompson JD, Gibson TJ, Plewniak F, Jeanmougin F, Higgins DG. The CLUSTAL_X windows interface: flexible strategies for multiple sequence alignment aided by quality analysis tools. Nucleic Acids Res 1997;25:4876-82. 
16. Kimura M. A simple method for estimating evolutionary rates of base substitutions through comparative studies of nucleotide sequences. J Mol Evol 1980;16:111-20.

17. Felsenstein J. Confidence limits on phylogenies: an approach using the bootstrap. Evolution 1985;39:783-91.
18. Johnson VA, Brun-Vézinet F, Clotet B, Günthard HF, Kuritzkes DR, Pillay D, et al. Update of the drug resistance mutations in HIV-1: December 2008. Top HIV Med 2008;16:138-45.

19. McCutchan FE. Global epidemiology of HIV. J Med Virol 2006;78:S7-12. 